\title{
Identification of Potential Novel Inhibitors for Nipah Virus - An in silico Approach
}

\author{
T. Raja $^{1}$, P. Ravikumar ${ }^{1}$, M. R. Srinivasan ${ }^{2}$, K. Vijayarani ${ }^{\text {* }}$ and K. Kumanan ${ }^{1}$ \\ ${ }^{1}$ Bioinformatics Centre and ARIS Cell, ${ }^{2}$ Department of Veterinary Pharmacology and \\ Toxicology, Madras Veterinary College, Tamil Nadu Veterinary and Animal Sciences \\ University, Chennai - 600 007, Tamil Nadu, India \\ *Corresponding author
}

\section{A B S T R A C T}

\begin{tabular}{l} 
K e y w o r d s \\
Nipah Virus, \\
ADMET prediction, \\
Toxicity studies, \\
$\begin{array}{l}\text { Docking, } \\
\text { Simulation study }\end{array}$ \\
Article Info \\
$\begin{array}{l}\text { Accepted: } \\
\text { 24 August } 2020 \\
\text { Available Online: } \\
\text { 10 September } 2020\end{array}$ \\
\hline
\end{tabular}

NipahVirus (NiV) is a zoonotic Virus which infects several animals and humans worldwide. Currently, there is no specific drug or vaccine to treat or control NiV infections. The present study is aimed to identify novel inhibitors of NiV from Indian medicinal plants. Glycoprotein was taken as target protein. Around 600 phytocompounds were taken from 10 Indian medicinal plants and subjected to Virtual Screening by using Libdock module from Discovery Studio 4.0. From these, the best 20 compounds were screened and evaluated for their Pharmacokinetic properties by using ADMETSAR and pKCSM tools. Toxicity studies were also performed using TOPKAT- Discovery Studio 4.0. Evaluated compounds were taken for individual docking. Molecular Dynamics (MD) simulation study was done by using GROMACS 5.1.2 to find the stability of the best interacted protein and ligand complex. From the results of this study, Neoandrographolide (Libdock score: 146.79), Hexahydrocurcumin (Libdock score: 146.501) and Nirphyllin (Libdock score: 141.896) showed better interaction with target protein. Hence, the present study concludes that the Neoandrographolide from Andrographis paniculata, Hexahydrocurcumin from Zingiber officinale and Nirphyllin from Phyllanthus amarus are having the potential ability to act as the best inhibitor for the target protein of NiV.

\section{Introduction}

Nipah virus belong to the genus Henipavirus of the family Paramyxoviridae and Nipah virus infections are of zoonotic nature (WHO, 2019). Encephalitis caused by Nipah virus is of significant public health importance and, based on the diffusion patterns and mortality rates, two different strains of Nipah virus (NiV) namely, NiV Malaysia (NiVM) and
NiV Bangladesh (NiVB) have been reported of which, NiVB is more pathogenic (Shimojima et al., 2006) than the other. Humans and many vertebrate animals are affected by NiV (Eaton et al., 2006) and NiV infections have significant economic importance in pig husbandry (WHO, 2019). The first outbreak of NiV was reported in the year 1998 in Kampung Sungai Nipah, Malaysia and subsequently in Bangladesh 
(Hsu et al., 2004). Neighbouring India reported two outbreaks in the year 2001 and 2007 in the state of West Bengal (Searo, 2019a). In South India, the first NiV outbreak was found in Kozhikode, Kerala during May 2018 with 17 human deaths (Searo, 2019b).

Though very few outbreaks of NiV have been reported in Asia, it resulted in human mortality (WHO, 2019). Though pigs play a role in the transmission of $\mathrm{NiV}$, involvement of pigs was not confirmed in the 1998-1999 outbreak in Malaysia. There has also been a strong evidence of human-to-human transmission of $\mathrm{NiV}$ in few of the Indian and Bangladesh outbreaks. In Bangladesh, 161 people were reported to have been died by NiV infection (Searo, 2019a).

Fruit bats (Flying foxes) are reported to be the natural wildlife reservoir of $\mathrm{NiV}$ which transmit the virus to both humans and animals. The Indian Flying Fox (Pteropus giganteus) and short-nosed Indian fruit bat (Cynopterus sphinx) are the mostly prevalent species in South Asia which act as main carriers for $\mathrm{NiV}$ (Bishop et al., 2008). NiV has been found in urine, kidney and uterus of infected wild bats and the virus has also been found in fruits or juice like date palm sap contaminated with the urine or saliva of bat. Polluted drinking water, abandoned bat foetuses or other fluids/tissues of parturition have been reported as other source of $\mathrm{NiV}$ infections (WOAH, 2009).

Consumption of fruits or fruit products like contaminated raw date palm sap are the main causes for $\mathrm{NiV}$ outbreaks in India and Bangladesh (Luby et al., 2012) apart from those working in trees (Montgomery et al., 2008). In humans, symptoms of NiV infection generally include headache, fever, sore throat, myalgia and vomiting followed by dizziness, altered consciousness, and neurological signs like acute encephalitis. Atypical pneumonia and respiratory problems are also noticed in few instances with seizures leading to coma (WHO, 2019).

In domestic animals, pigs may be symptomless, but other animals might show acute febrile illness, laboured breathing, and neurological signs like trembling, twitching and muscle spasms (Nor et al., 2000).

Understanding the molecular structure of the pathogens is essential in designing treatment and implementing control strategies. $\mathrm{NiV}$ genome consists six coding regions and several non-coding regions. The six coding regions include, Nucleoprotein $(\mathrm{N})$, Large (L) protein, Phosphoprotein (P), Matrix (M) protein, Fusion $(\mathrm{F})$ protein and Glycoprotein (G) wherein, nucleoprotein is used to make nucleocapsid and acts as a model for replication and transcription of the virus.

In addition, RNA dependent RNA polymerase accelerates this protein (Wang et al., 2001). Fusion protein is responsible for membrane fusion (Horvath et al., 1992) and Glycoprotein is involved in fusion and attachment (Moller-Tank and Maury, 2015, Mehmood et al., 2014, Moll et al., 2004). In the present study, Glycoprotein was taken as target protein.

As of now, there are no specific drugs and vaccines available for the treatment and control of $\mathrm{NiV}$ infections, necessitating the need for focused research in developing vaccines and identifying possible therapeutic molecules. Use of medicinal plants is one of the priority areas in combating viral diseases and hence, the present study is designed to identify potential novel inhibitors of $\mathrm{NiV}$ from Indian medicinal plants through Structure based virtual screening, Pharmacokinetic, Docking and Simulation studies. 
Materials and Methods

\section{Selection and retrieval of 3D structures of target protein}

In the present study, Glycoprotein (PDB ID: 3D11) was selected as target protein. The 3D structure of this protein was downloaded from the PDB database.

\section{Selection and retrieval of 3D structures of ligands}

Around 600 compounds were selected from ten medicinal plants namely, Zingiber officinale, Punica granatum, Phyllanthus amarus, Ocimum basilicum, Momordica charantia, Hypericum perforatum, Curcuma longa, Centella asiatica, Azadirachta indica and Andrographis paniculata using IMPPAT database (Mohanraj et al., 2018), of which 250 compounds were shortlisted based on the Lipinski Rule of Five. Further, 3D structures of ligands were retrieved from PubChem database.

\section{Virtual screening and docking}

The 250 phytocompounds selected were subjected to virtual screening with target protein and the results were analyzed. Based on the analysis, the best compound was subjected to docking with target protein using LibDock module of Discovery studio 4.0 wherein higher LibDock scores indicated better affinity.

\section{Pharmacokinetic and Toxicity studies}

Compounds having the best LibDock score were evaluated for pharmacokinetic properties using ADMETSAR and pKCSM tools. Toxicity studies were also performed for the best compounds using TOPKAT- Discovery Studio 4.0 and the results were analyzed.

\section{Simulation studies}

Molecular Dynamics (MD) simulation was employed to investigate the stability of protein-ligand complex generated from the Discovery Studio 4.0 docking procedures. Gromacs 5.1.2 package was employed to perform MD simulation. Before MD simulation, Gromos 9643a1 force field was used for the protein coordinate parameters and topology. The ligand topology and initial geometries of the ligand were taken from PRODRG web server (Schuttelkopf and Van Aalten, 2004). The systems were designed after merging the coordinates and topology parameters of both the protein and ligand. TIP3P water model was used to solvate the system in a dodecahedron box and counter ions were used to neutralize the system. Steepest descent algorithm was used for energy minimization for 1000 steps. When the upper limit of the force was lower than 1000 $\mathrm{kJ} / \mathrm{mol}$, bad contacts and steric clashes of protein-ligand complexes were removed. Subsequently, protein-ligand and solvent-ions groups were formed in each minimized system for avoiding subside and it was taken for equilibration. Two components were involved in the equilibration of each system. With the help of Berendsen thermostat algorithm, the first equilibration was done at constant volume (NVT) for 100ps at constant temperature of $300 \mathrm{~K}$. According to the algorithm of Parrinello- Rahmanbarostat and LINCS, the second equilibration was performed for $100 \mathrm{ps}$ at constant pressure (NPT) of 1 bar. Electrostatic interactions were examined by Particle Mesh Ewald method (Essmann et al., 1995). Thus, after completing all the optimization procedures, the simulation was done for $10 \mathrm{~ns}(10000 \mathrm{ps})$ to evaluate the stability. Finally, Root Mean Square Deviation (RMSD), Root Mean Square Fluctuations (RMSF) and Radius of Gyration $(\operatorname{Rg})$ were analyzed using the GROMACS comments g_rmsd, g_rmsf and 
g_gyrate, respectively (Van Der Spoel et al., 2005).

\section{Results and Discussion}

\section{Selection and retrieval of 3D structures of target protein and ligands}

As there are no specific treatment or vaccines for $\mathrm{NiV}$ infection, increasing the public awareness about NiV infections, and identifying the therapeutic and vaccine candidates could be the possible solutions to prevent future NiV infections. Ribavirin, the drug used during $\mathrm{NiV}$ infections will be useful only in alleviating nausea, vomiting and convulsions (Chong et al., 2001). Hence, in this study, we tried to identify potential inhibitors of NiV from Indian medicinal plants using in silico methods.

The 3D structure of Glycoprotein (PDB ID: 3D11) was retrieved from PDB database and the 3D structures of all the ligands were taken from PubChem database and their CID numbers were noted.

\section{Virtual screening and docking studies}

In our study, the target protein (glycoprotein) of $\mathrm{NiV}$ was subjected to virtual screening and docking with phytocompounds of ten Indian medicinal plants and the best results obtained are shown in Table 1. Further, the 2D and 3D interaction of target protein with the best ligands are shown in Fig. (1-6).

NiV Glycoprotein was docked with phytocompounds of Indian medicinal plants (Table 1 and Fig. (1-6). 10 compounds were identified as good inhibitor of which, Neoandrographolide from Andrographis paniculata showed the best LibDock score of 146.79 and formed 14 hydrogen bonds with ASP 219, PRO 220, HIS 281, CYS 282, GLY 352, GLU 505 and GLY 506. Here the nitrogen atom in the amino acid residues of
GLY 506 and GLY 352, spared one hydrogen atom to the oxygen atom of the ligand to form hydrogen bond between ligand and amino acid residues of the target protein. In addition, oxygen atom of CYS 282, GLY 352, PRO 220 and ASP 219 residues accepted hydrogen from the ligand Neoandrographolide to form hydrogen bond. Further, carbon atom in the GLU 505 spared hydrogen to the oxygen atom of the ligand to form hydrogen bond.

Similarly, nitrogen atom of the HIS 281 accepted hydrogen from carbon and oxygen atom of the ligand to form 2 hydrogen bonds. Thus, NiV glycoprotein interacted with neoandrographolide to form hydrogen bonds.

In addition, the interaction of glycoprotein with hexahydrocurcumin from Zingiber officinale gave the best LibDock score of 146.5 and formed 7 hydrogen bonds with CYS 282, LYS 560 and ASP 219, where CYS 282 and LYS 560 released hydrogen atom to make hydrogen bonds. ASP 219 received hydrogen atom to make hydrogen bonds. Nirphyllin from Phyllanthus amarus also showed better LibDock score (141.9) with 15 hydrogen bonds, where GLN 559, LYS 560, HIS 281, PRO 353, GLU 579 spared hydrogen atom to form hydrogen bonds and TYR 351, ASP 302, PRO 220, CYS 282, GLU 579 accepted hydrogen atom to form hydrogen bonds with ligands. Besides, among the ten inhibitors, Nimbidic acid from Azadirachta indica showed lowest Libdock score of 131.505 with 5 hydrogen bonds.

Though some of the earlier attempts have shortlisted around five compounds from fewer proteins (Ali et al., 2018, Archana et al., 2018), we targeted Glycoprotein. Our results identified Neoandrographolide from Andrographis paniculata, Hexahydrocurcumin from Zingiber officinale and Nirphyllin from Phyllanthus amarus as potential inhibitor of $\mathrm{NiV}$. 


\section{Pharmacokinetic properties}

Phytocompounds having good LibDock score with the target protein of $\mathrm{NiV}$ were evaluated for pharmacokinetic properties using ADMETSAR and pKCSM and the results are compiled and presented in Table 2. In the absorption parameters, all the molecules were found to have human intestinal absorption properties in ADMETSAR software. This is supported by the $\mathrm{CaCo} 2$ permeability prediction values as well. However, only one molecule, Niranthin was found positive for oral bioavailability but the other molecules showed negativity in bioavailability parameter which could be due to the first pass metabolism. This prediction could be weighed with caution since the degree of first pass metabolism is not predicted.

Table.1 Interaction of glycoprotein with phytocompounds

\begin{tabular}{|c|c|c|c|c|c|c|c|}
\hline \multicolumn{8}{|c|}{ Glycoprotein } \\
\hline $\begin{array}{l}\text { S. } \\
\text { No. }\end{array}$ & $\begin{array}{l}\text { PubChem } \\
\text { (CID) }\end{array}$ & Compound Name & Plant Name & $\begin{array}{l}\text { LibDock } \\
\text { Score }\end{array}$ & $\begin{array}{l}\text { Number } \\
\text { of } H- \\
\text { bond }\end{array}$ & $\begin{array}{c}\text { Interacting residues } \\
(\text { (D-H...A) }\end{array}$ & $\begin{array}{l}\text { Bond } \\
\text { Length } \\
\text { (A) }\end{array}$ \\
\hline 1 & 9848024 & Neoandrographolide & $\begin{array}{l}\text { Andrographis } \\
\text { paniculata }\end{array}$ & 146.79 & 14 & $\begin{array}{l}\text { GLY } 352(\mathrm{~N}-\mathrm{H} \ldots \mathrm{O}) \\
\text { GLY } 506(\mathrm{~N}-\mathrm{H} \ldots \mathrm{O}) \\
\text { HIS } 281(\mathrm{~N} \ldots \mathrm{H}-\mathrm{O}) \\
\text { CYS } 282(\mathrm{O} \ldots \mathrm{H}-\mathrm{O}) \\
\text { GLY } 352(\mathrm{O} \ldots \mathrm{H}-\mathrm{O}) \\
\text { CYS } 282(\mathrm{O} \ldots \mathrm{H}-\mathrm{O}) \\
\text { GLY } 352(\mathrm{O} \ldots \mathrm{H}-\mathrm{O}) \\
\text { GLU } 505(\mathrm{C}-\mathrm{H} \ldots \mathrm{O}) \\
\text { HIS } 281(\mathrm{~N} \ldots \mathrm{H}-\mathrm{C}) \\
\text { PRO } 220(\mathrm{O} \ldots \mathrm{H}-\mathrm{C}) \\
\text { CYS } 282(\mathrm{O} \ldots \mathrm{H}-\mathrm{C}) \\
\text { ASP } 219(\mathrm{O} \ldots \mathrm{H}-\mathrm{C}) \\
\text { ASP } 219(\mathrm{O} \ldots \mathrm{H}-\mathrm{C}) \\
\text { PRO } 220(\mathrm{O} \ldots \mathrm{H}-\mathrm{C})\end{array}$ & $\begin{array}{l}2.08 \\
2.10 \\
2.01 \\
1.95 \\
2.69 \\
1.70 \\
2.75 \\
2.18 \\
2.58 \\
2.63 \\
2.44 \\
2.95 \\
2.22 \\
2.44\end{array}$ \\
\hline 2 & 5318039 & Hexahydrocurcumin & $\begin{array}{l}\text { Zingiber } \\
\text { officinale }\end{array}$ & 146.501 & 7 & $\begin{array}{l}\text { CYS } 282(\mathrm{~N}-\mathrm{H} . . \mathrm{O}) \\
\text { LYS } 560(\mathrm{~N}-\mathrm{H} \ldots \mathrm{O}) \\
\text { LYS } 560(\mathrm{~N}-\mathrm{H} \ldots \mathrm{O}) \\
\text { ASP } 219(\mathrm{O} \ldots \mathrm{H}-\mathrm{O}) \\
\text { ASP } 219(\mathrm{O} \ldots \mathrm{H}-\mathrm{O}) \\
\text { LYS } 560(\mathrm{C}-\mathrm{H} \ldots \mathrm{O}) \\
\text { ASP } 219(\mathrm{O} \ldots \mathrm{H}-\mathrm{C})\end{array}$ & $\begin{array}{l}1.95 \\
2.14 \\
1.87 \\
2.03 \\
1.82 \\
2.94 \\
2.30\end{array}$ \\
\hline 3 & 5491556 & Nirphyllin & $\begin{array}{l}\text { Phyllanthus } \\
\text { amarus }\end{array}$ & 141.896 & 15 & $\begin{array}{l}\text { GLN } 559(\mathrm{~N}-\mathrm{H} . . \mathrm{O}) \\
\text { GLN } 559(\mathrm{~N}-\mathrm{H} \ldots \mathrm{O}) \\
\text { LYS } 560(\mathrm{~N}-\mathrm{H} \ldots \mathrm{O}) \\
\text { HIS } 281(\mathrm{C}-\mathrm{H} \ldots \mathrm{O}) \\
\text { PRO } 353(\mathrm{C}-\mathrm{H} \ldots \mathrm{O}) \\
\text { TYR } 351(\mathrm{O} \ldots \mathrm{H}-\mathrm{C}) \\
\text { ASP } 302(\mathrm{O} \ldots \mathrm{H}-\mathrm{C}) \\
\text { TYR } 351(\mathrm{O} \ldots \mathrm{H}-\mathrm{C}) \\
\text { ASP } 302(\mathrm{O} \ldots \mathrm{H}-\mathrm{C}) \\
\text { ASP } 302(\mathrm{O} \ldots \mathrm{H}-\mathrm{C}) \\
\text { PRO } 220(\mathrm{O} \ldots \mathrm{H}-\mathrm{C}) \\
\text { CYS } 282(\mathrm{O} \ldots \mathrm{H}-\mathrm{C}) \\
\text { GLU } 579(\mathrm{O} \ldots \mathrm{H}-\mathrm{C}) \\
\text { GLU } 579(\mathrm{O} \ldots \mathrm{H}-\mathrm{C}) \\
\text { GLU } 579(\mathrm{C}-\mathrm{H} \ldots \mathrm{O})\end{array}$ & $\begin{array}{l}2.15 \\
1.67 \\
2.89 \\
2.73 \\
2.70 \\
2.54 \\
2.96 \\
2.58 \\
2.51 \\
2.56 \\
2.67 \\
2.53 \\
2.55 \\
2.63 \\
2.96\end{array}$ \\
\hline
\end{tabular}


Int.J.Curr.Microbiol.App.Sci (2020) 9(9): 3377-3390

\begin{tabular}{|c|c|c|c|c|c|c|c|}
\hline 4 & 9796792 & $\begin{array}{l}\text { Tetrahydrobisdemethox } \\
\text { ycurcumin }\end{array}$ & Curcuma longa & 136.915 & 6 & $\begin{array}{l}\text { LYS } 560(\mathrm{~N}-\mathrm{H} \ldots \mathrm{O}) \\
\text { LYS } 560(\mathrm{~N}-\mathrm{H} \ldots \mathrm{O}) \\
\text { GLY } 506(\mathrm{O} \ldots \mathrm{H}-\mathrm{O}) \\
\text { ASP } 219(\mathrm{O} \ldots \mathrm{H}-\mathrm{O}) \\
\text { ASP } 219(\mathrm{C}-\mathrm{H} \ldots \mathrm{O}) \\
\text { LYS } 560(\mathrm{C}-\mathrm{H} \ldots \mathrm{O})\end{array}$ & $\begin{array}{l}1.66 \\
1.72 \\
1.74 \\
1.80 \\
2.46 \\
2.41\end{array}$ \\
\hline 5 & 5318568 & Isogingerenone $\mathrm{B}$ & $\begin{array}{l}\text { Zingiber } \\
\text { officinale }\end{array}$ & 136.275 & 7 & $\begin{array}{l}\text { GLY } 352(\mathrm{~N}-\mathrm{H} \ldots \mathrm{O}) \\
\text { TYR } 508(\mathrm{~N}-\mathrm{H} \ldots \mathrm{O}) \\
\text { GLN } 490(\mathrm{O} \ldots \mathrm{H}-\mathrm{O}) \\
\text { CYS } 282(\mathrm{O} \ldots \mathrm{H}-\mathrm{O}) \\
\text { GLU } 505(\mathrm{C}-\mathrm{H} \ldots \mathrm{O}) \\
\text { VAL } 507(\mathrm{C}-\mathrm{H} \ldots \mathrm{O}) \\
\text { ASP } 219(\mathrm{O} \ldots \mathrm{H}-\mathrm{C})\end{array}$ & $\begin{array}{l}2.03 \\
1.98 \\
2.32 \\
1.78 \\
2.74 \\
2.88 \\
3.00\end{array}$ \\
\hline 6 & 5281775 & Gingerenone A & $\begin{array}{l}\text { Zingiber } \\
\text { officinale }\end{array}$ & 135.903 & 11 & $\begin{array}{l}\text { GLY } 352(\mathrm{~N}-\mathrm{H} \ldots \mathrm{O}) \\
\text { TYR } 508(\mathrm{~N}-\mathrm{H} \ldots \mathrm{O}) \\
\text { TRP } 504(\mathrm{O} \ldots \mathrm{H}-\mathrm{O}) \\
\text { CYS } 282(\mathrm{O} \ldots \mathrm{H}-\mathrm{O}) \\
\text { GLY } 352(\mathrm{O} \ldots \mathrm{H}-\mathrm{O}) \\
\text { HIS } 281(\mathrm{C}-\mathrm{H} \ldots \mathrm{O}) \\
\text { GLU } 505(\mathrm{C}-\mathrm{H} \ldots \mathrm{O}) \\
\text { VAL } 507(\mathrm{C}-\mathrm{H} \ldots \mathrm{O}) \\
\text { GLY } 506(\mathrm{O} \ldots \mathrm{H}-\mathrm{C}) \\
\text { GLN } 490(\mathrm{O} \ldots \mathrm{H}-\mathrm{C}) \\
\text { PRO } 220(\mathrm{O} \ldots \mathrm{H}-\mathrm{C})\end{array}$ & $\begin{array}{l}2.18 \\
2.07 \\
2.68 \\
1.85 \\
2.76 \\
2.92 \\
2.40 \\
2.68 \\
2.55 \\
2.35 \\
2.51\end{array}$ \\
\hline 7 & 13989915 & Niranthin & $\begin{array}{l}\text { Phyllanthus } \\
\text { amarus }\end{array}$ & 133.595 & 6 & $\begin{array}{l}\text { THR } 218(\mathrm{C}-\mathrm{H} . . . \mathrm{O}) \\
\text { HIS } 281(\mathrm{C}-\mathrm{H} \ldots \mathrm{O}) \\
\text { ASP } 219(\mathrm{O} \ldots \mathrm{H}-\mathrm{C}) \\
\text { CYS } 282(\mathrm{O} \ldots \mathrm{H}-\mathrm{C}) \\
\text { CYS } 240(\mathrm{O} \ldots \mathrm{H}-\mathrm{C}) \\
\text { CYS } 240(\mathrm{O} \ldots \mathrm{H}-\mathrm{C})\end{array}$ & $\begin{array}{l}2.47 \\
2.43 \\
2.98 \\
2.42 \\
2.49 \\
2.36\end{array}$ \\
\hline 8 & 5317593 & Gingerenone $\mathrm{C}$ & $\begin{array}{l}\text { Zingiber } \\
\text { officinale }\end{array}$ & 132.865 & 4 & $\begin{array}{l}\text { LYS } 560(\mathrm{~N}-\mathrm{H} \ldots \mathrm{O}) \\
\text { GLY } 506(\mathrm{O} \ldots \mathrm{H}-\mathrm{O}) \\
\text { ASP } 219(\mathrm{O} \ldots \mathrm{H}-\mathrm{O}) \\
\text { LYS } 560(\mathrm{C}-\mathrm{H} \ldots \mathrm{O})\end{array}$ & $\begin{array}{l}2.33 \\
1.99 \\
1.78 \\
2.26\end{array}$ \\
\hline 9 & 5469424 & Demethoxycurcumin & Curcuma longa & 132.799 & 4 & $\begin{array}{l}\text { GLN } 559(\mathrm{~N}-\mathrm{H} \ldots \mathrm{O}) \\
\text { LYS } 560(\mathrm{~N}-\mathrm{H} \ldots \mathrm{O}) \\
\text { SER } 232(\mathrm{O} \ldots \mathrm{H}-\mathrm{O}) \\
\text { HIS } 281(\mathrm{C}-\mathrm{H} \ldots \mathrm{O})\end{array}$ & $\begin{array}{l}2.45 \\
1.67 \\
2.32 \\
2.42\end{array}$ \\
\hline 10 & $29803-85-8$ & Nimbidic Acid & $\begin{array}{l}\text { Azadirachta } \\
\text { indica }\end{array}$ & 131.505 & 5 & $\begin{array}{l}\text { ASP } 219(\mathrm{~N}-\mathrm{H} \ldots \mathrm{O}) \\
\text { ARG } 248(\mathrm{~N}-\mathrm{H} \ldots \mathrm{O}) \\
\text { ASP } 219(\mathrm{C}-\mathrm{H} \ldots \mathrm{O}) \\
\text { ASP } 219(\mathrm{O} \ldots \mathrm{H}-\mathrm{C}) \\
\text { ASP } 219(\mathrm{O} \ldots \mathrm{H}-\mathrm{C})\end{array}$ & $\begin{array}{l}2.74 \\
2.63 \\
2.75 \\
2.62 \\
2.78\end{array}$ \\
\hline
\end{tabular}


Table.2 Pharmacokinetic properties of phytocompounds

\begin{tabular}{|c|c|c|c|c|c|c|c|c|c|c|c|c|c|c|c|c|}
\hline \multirow[t]{2}{*}{$\begin{array}{l}\text { S. } \\
\text { No. }\end{array}$} & \multirow[t]{2}{*}{$\begin{array}{l}\text { Name of the } \\
\text { Phytocompound }\end{array}$} & \multicolumn{3}{|c|}{ Absorption $^{1,2}$} & \multicolumn{5}{|c|}{ Distribution $^{1,2}$} & \multicolumn{5}{|c|}{$\begin{array}{c}\text { Metabolism - CYP inhibition (-/+) or } \\
\text { substrate }(\mathrm{S})^{1,2}\end{array}$} & \multicolumn{2}{|c|}{ Excretion $^{2}$} \\
\hline & & HIA & HOB & $\mathrm{Cc} 2$ & PPB & PgpI & PgpS & BBB & VD & $1 \mathrm{~A} 2$ & $2 D 6$ & $2 \mathrm{C} 9$ & $2 \mathrm{C19}$ & 3A4 & TC & $\begin{array}{l}\text { Renal } \\
\text { OCT2 }\end{array}$ \\
\hline 1 & Neoandrographolide & + & - & - & 0.83 & - & - & - & -1.08 & - & - & - & - & $-/ \mathrm{S}$ & 0.94 & No \\
\hline 2 & Hexahydrocurcumin & + & - & - & 0.73 & - & - & - & 0.32 & + & $-/ S$ & - & + & $-/ \mathrm{S}$ & 0.31 & No \\
\hline 3 & Nirphyllin & + & - & + & 1.10 & + & - & + & -0.18 & - & $-/ S$ & + & + & $+/ \mathrm{S}$ & 0.63 & No \\
\hline 4 & $\begin{array}{l}\text { Tetrahydrobisdemethoxy } \\
\text { curcumin }\end{array}$ & + & - & - & 0.55 & - & - & - & 0.32 & - & - & + & - & + & 0.22 & No \\
\hline 5 & Isogingerenone B & + & - & - & 0.92 & + & - & + & 0.04 & + & - & - & + & $-/ \mathrm{S}$ & 0.27 & No \\
\hline 6 & Gingerenone A & + & - & - & 0.85 & + & - & + & 0.02 & + & - & + & + & - & 0.23 & No \\
\hline 7 & Niranthin & + & + & + & 0.97 & + & - & + & -0.10 & - & $+/ \mathrm{S}$ & + & + & $+/ \mathrm{S}$ & 0.47 & No \\
\hline 8 & Gingerenone $\mathrm{C}$ & + & - & - & 0.80 & - & - & + & 0.23 & + & - & + & + & $-/ \mathrm{S}$ & 0.21 & No \\
\hline 9 & Demethoxycurcumin & + & - & - & 0.72 & - & - & - & 0.10 & + & - & + & + & - & 0.04 & No \\
\hline 10 & Nimbidic acid & + & - & - & 0.81 & - & + & + & -0.26 & - & - & - & - & $-/ \mathrm{S}$ & 0.32 & No \\
\hline
\end{tabular}

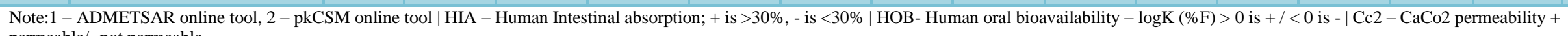
permeable/- not permeable

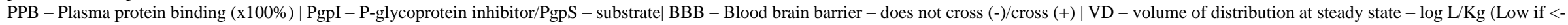
0.15 and high if $>0.45$ )

CYP- Cytochrome P450 inhibitor (+ yes/ - no) or Substrate

$\mathrm{TC}$ - Total clearance $(\log \mathrm{ml} / \mathrm{min} / \mathrm{kg}$ ); higher the value higher is the clearance and vice versa | Renal OCT2- organic cationic transporter 2 - NO (not a substrate) /Sub (substrate) 
Table.3 Toxicity prediction of phytocompounds

\begin{tabular}{|c|c|c|c|c|c|c|c|c|c|c|}
\hline $\begin{array}{l}\text { S. } \\
\text { No } \\
\text {. }\end{array}$ & $\begin{array}{l}\text { Phyto compounds \& } \\
\text { PubChem CID }\end{array}$ & $\begin{array}{c}\text { TOPKAT Rat } \\
\text { Female NTP } \\
\text { Prediction }\end{array}$ & $\begin{array}{l}\text { TOPKAT } \\
\text { Rat Male } \\
\text { NTP } \\
\text { Prediction }\end{array}$ & $\begin{array}{c}\text { TOPKAT } \\
\text { Mouse Female } \\
\text { FDA None vs } \\
\text { Carcinogen } \\
\text { Prediction }\end{array}$ & $\begin{array}{c}\text { TOPKAT } \\
\text { Mouse Male } \\
\text { FDA None vs } \\
\text { Carcinogen } \\
\text { Prediction }\end{array}$ & $\begin{array}{l}\text { TOPKAT } \\
\text { Ames } \\
\text { Prediction }\end{array}$ & $\begin{array}{l}\text { TOPKAT } \\
\text { Skin } \\
\text { Irritancy }\end{array}$ & $\begin{array}{c}\text { TOPKAT } \\
\text { Skin } \\
\text { Sensitization }\end{array}$ & $\begin{array}{l}\text { TOPKAT } \\
\text { Ocular } \\
\text { Irritancy }\end{array}$ & $\begin{array}{c}\text { TOPKAT } \\
\text { Aerobic Bio } \\
\text { degradability } \\
\text { Prediction }\end{array}$ \\
\hline 1 & $\begin{array}{l}\text { Neoandrographolide } \\
(9848024)\end{array}$ & $\begin{array}{l}\text { Non- } \\
\text { Carcinogen }\end{array}$ & $\begin{array}{l}\text { Non- } \\
\text { Carcinogen }\end{array}$ & Non-Carcinogen & $\begin{array}{l}\text { Non- } \\
\text { Carcinogen }\end{array}$ & Non-Mutagen & Moderate & None & None & Degradable \\
\hline 2 & $\begin{array}{l}\text { Hexahydrocurcumin } \\
(5318039)\end{array}$ & $\begin{array}{l}\text { Non- } \\
\text { Carcinogen }\end{array}$ & $\begin{array}{l}\text { Non- } \\
\text { Carcinogen }\end{array}$ & Non-Carcinogen & $\begin{array}{l}\text { Non- } \\
\text { Carcinogen }\end{array}$ & Non-Mutagen & None & Strong & Moderate & $\begin{array}{l}\text { Non- } \\
\text { Degradable }\end{array}$ \\
\hline 3 & $\begin{array}{l}\text { Nirphyllin } \\
(5491556)\end{array}$ & $\begin{array}{l}\text { Non- } \\
\text { Carcinogen }\end{array}$ & $\begin{array}{l}\text { Non- } \\
\text { Carcinogen }\end{array}$ & Non-Carcinogen & $\begin{array}{l}\text { Non- } \\
\text { Carcinogen }\end{array}$ & Non-Mutagen & Mild & Strong & Mild & Degradable \\
\hline 4 & $\begin{array}{l}\text { Tetrahydrobisdemethoxyc } \\
\text { urcumin (9796792) }\end{array}$ & $\begin{array}{l}\text { Non- } \\
\text { Carcinogen }\end{array}$ & $\begin{array}{l}\text { Non- } \\
\text { Carcinogen }\end{array}$ & Carcinogen & $\begin{array}{l}\text { Non- } \\
\text { Carcinogen }\end{array}$ & Non-Mutagen & None & Strong & Severe & $\begin{array}{l}\text { Non- } \\
\text { Degradable }\end{array}$ \\
\hline 5 & $\begin{array}{l}\text { Isogingerenone B } \\
(5318568)\end{array}$ & $\begin{array}{l}\text { Non- } \\
\text { Carcinogen }\end{array}$ & $\begin{array}{l}\text { Non- } \\
\text { Carcinogen }\end{array}$ & Non-Carcinogen & $\begin{array}{l}\text { Non- } \\
\text { Carcinogen }\end{array}$ & Non-Mutagen & Mild & Strong & Severe & $\begin{array}{l}\text { Non- } \\
\text { Degradable }\end{array}$ \\
\hline 6 & $\begin{array}{l}\text { Gingerenone A } \\
(5281775)\end{array}$ & $\begin{array}{l}\text { Non- } \\
\text { Carcinogen }\end{array}$ & $\begin{array}{l}\text { Non- } \\
\text { Carcinogen }\end{array}$ & Non-Carcinogen & $\begin{array}{l}\text { Non- } \\
\text { Carcinogen }\end{array}$ & Non-Mutagen & Mild & Strong & Severe & $\begin{array}{l}\text { Non- } \\
\text { Degradable }\end{array}$ \\
\hline 7 & $\begin{array}{l}\text { Niranthin } \\
(13989915)\end{array}$ & $\begin{array}{l}\text { Non- } \\
\text { Carcinogen }\end{array}$ & $\begin{array}{l}\text { Non- } \\
\text { Carcinogen }\end{array}$ & Non-Carcinogen & $\begin{array}{l}\text { Non- } \\
\text { Carcinogen }\end{array}$ & Non-Mutagen & Moderate & Strong & Mild & Degradable \\
\hline 8 & $\begin{array}{l}\text { Gingerenone C } \\
(5317593)\end{array}$ & $\begin{array}{l}\text { Non- } \\
\text { Carcinogen }\end{array}$ & $\begin{array}{l}\text { Non- } \\
\text { Carcinogen }\end{array}$ & Non-Carcinogen & $\begin{array}{l}\text { Non- } \\
\text { Carcinogen }\end{array}$ & Non-Mutagen & Mild & Strong & Severe & $\begin{array}{l}\text { Non- } \\
\text { Degradable }\end{array}$ \\
\hline 9 & $\begin{array}{l}\text { Demethoxycurcumin } \\
\text { (5469424) }\end{array}$ & Carcinogen & $\begin{array}{l}\text { Non- } \\
\text { Carcinogen }\end{array}$ & Non-Carcinogen & Carcinogen & Non-Mutagen & Mild & Strong & Mild & Degradable \\
\hline 10 & $\begin{array}{l}\text { Nimbidic Acid } \\
(29803-85-8)\end{array}$ & $\begin{array}{l}\text { Non- } \\
\text { Carcinogen }\end{array}$ & $\begin{array}{l}\text { Non- } \\
\text { Carcinogen }\end{array}$ & Carcinogen & $\begin{array}{l}\text { Non- } \\
\text { Carcinogen }\end{array}$ & Non-Mutagen & Mild & None & Moderate & Degradable \\
\hline
\end{tabular}


Fig.1 3D interaction of Glycoprotein with Neoandrographolide

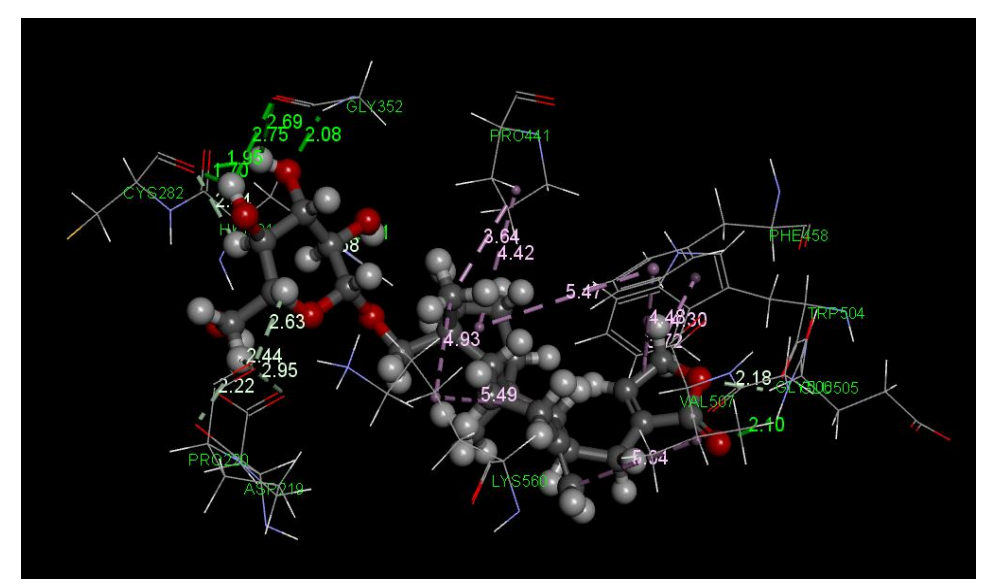

Fig.2 2D interaction of Glycoprotein with Neoandrographolide

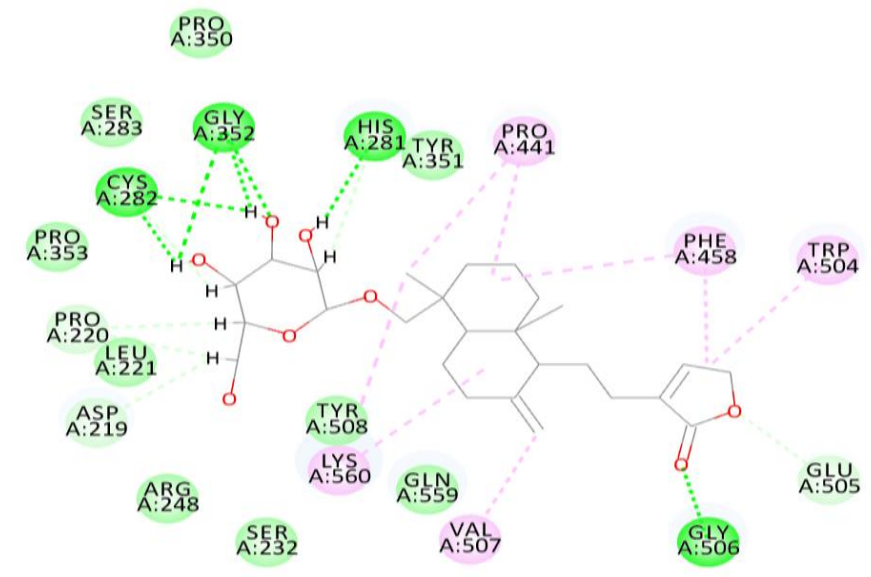

Fig.3 3D interaction of Glycoprotein with Hexahydrocurcumin

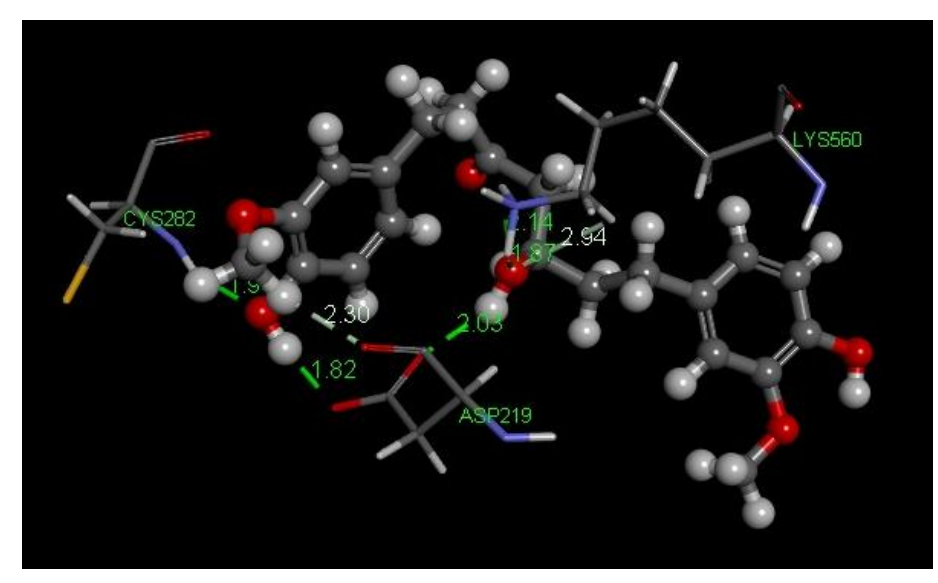


Fig.4 2D interaction of Glycoprotein with Hexahydrocurcumin

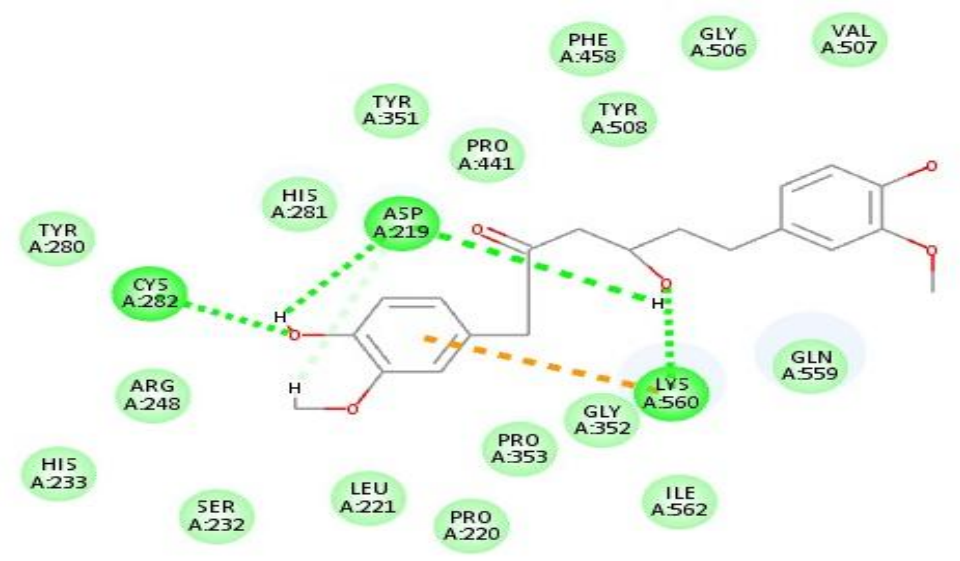

Fig.5 3D interaction of Glycoprotein with Nirphyllin

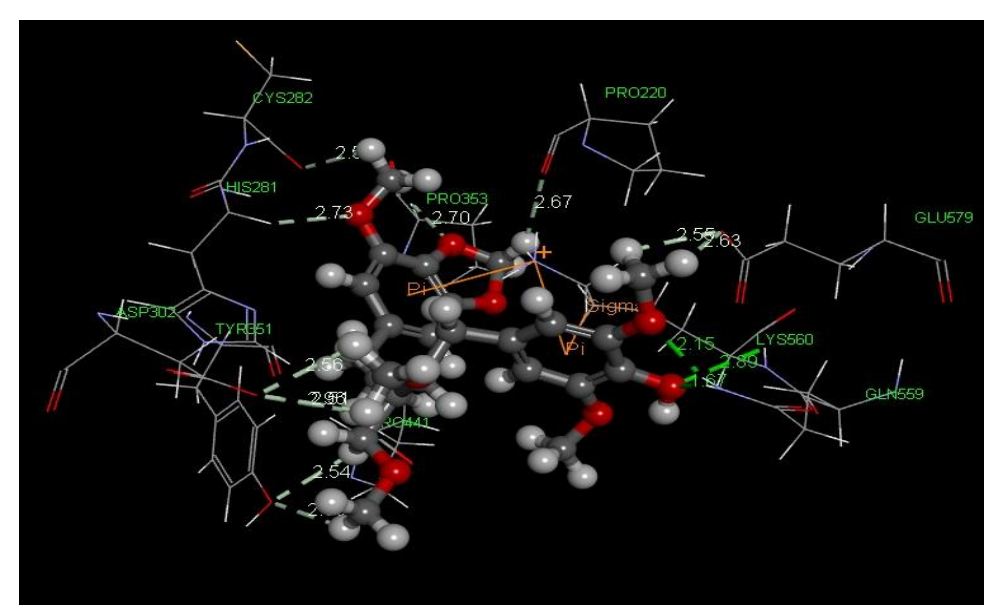

Fig.6 2D interaction of Glycoprotein with Nirphyllin

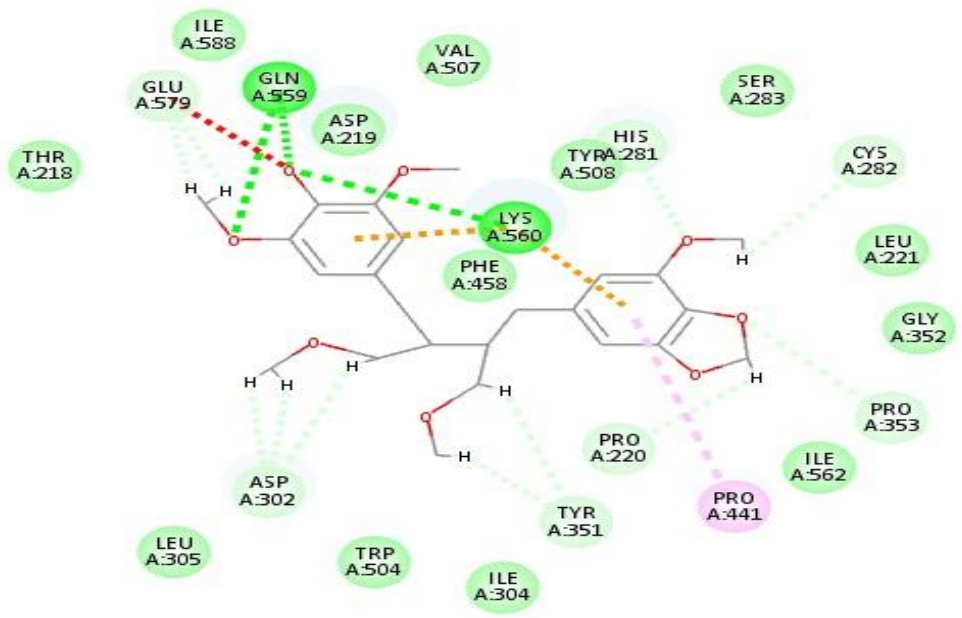


Fig.7 RMSD for the complex of Glycoprotein and Neoandrographolide

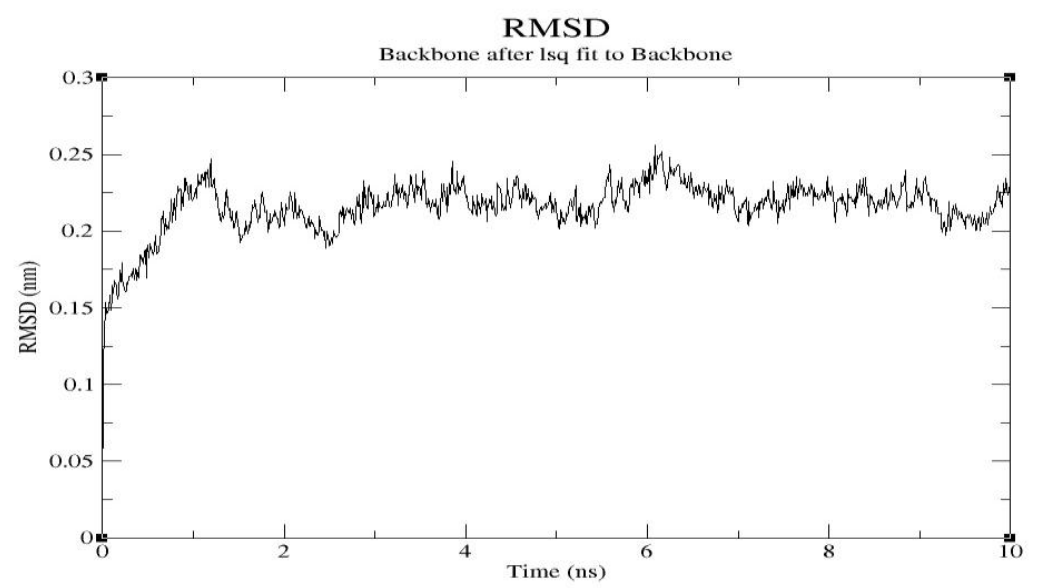

Fig.8 RMSF for the complex of Glycoprotein and Neoandrographolide

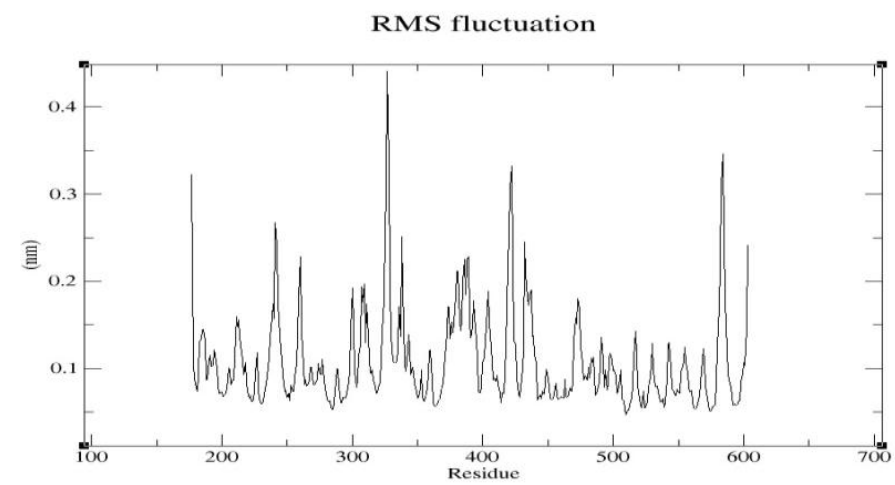

Fig.9 Radius of gyration for the complex of Glycoprotein and Neoandrographolide

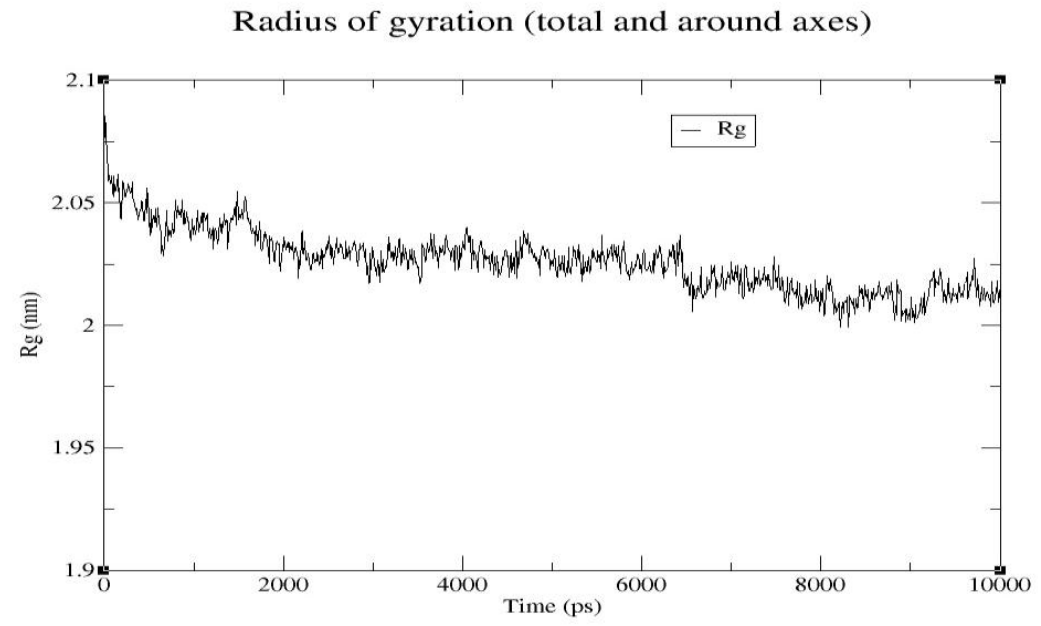


In case of distribution, all the compounds tested showed $50-100 \%$ plasma protein bindings, indicating the chances of longer duration action. Few compounds were found to be P-glycoprotein inhibitor which will not have any impact on its own intestinal absorption and only one compound, i.e., Nimbidic acid, showed as the substrate for Pgp which may have less intestinal absorption due to the possibility of efflux potential. All the compounds showed moderate to high volume of distribution.

In the metabolism profile, compounds showed both as CYP inhibitor and/or CYP susbstrate, hence the possibility of drug-drug interaction can happen with other drugs which requires the experimental studies to confirm the same.

In the excretion parameters, the total clearance was low $(<0.35 \mathrm{log} \mathrm{ml} / \mathrm{min} / \mathrm{kg})$ for 7 compounds and moderate to high $(>0.35)$ for the rest of the compounds. But, there were no compounds found to be substrate for renal OCT2 transporter. Among the 10 compounds, Neoandrographolide had highest docking score for which the molecular dynamics properties were studied.

Among the 10 compounds, Gingerenone showed favourable pharmacokinetics properties such as good human intestinal absorption, blood brain barrier crossing, moderate distribution, good plasma protein binding and less total clearance, indicating the possibility of longer duration of action.

Though Neoandrographolide showed less favourable pharmacokinetic properties, the binding property against the target proteins of Nipah virus was high. If experimentally proved, the pharmacokinetic parameters could be modified by the lead optimization strategy without affecting the activity of the compound.

\section{Toxicity prediction of phytocompounds}

In the toxicological parameters predicted using TOPKAT, all the compounds were found to be non-genotoxic, non-carcinogenic and had no hepatotoxic potentials. However, though there were indications for the skin sensitization, skin irritancy and ocular irritancy predicted for Neoandrographolide, Gingerenone A and C, these compound could still be subjected for further studies considering the positive aspects of these compounds and the possibilities of overcoming the drawbacks by proper precautionary measures (Table 3 ).

\section{Simulation studies}

Molecular dynamics simulation studies were done for the best interacted molecule for 10 ns using GROMACS 5.1.2. In the docking analysis, when glycoprotein was interacted with phytocompounds of medicinal plants, neoandrographolide showed the best Libdock score. Hence, the simulation study was done for the complex of glycoprotein and neoandrographolide to find their stability. RMSD, RMSF and Radius of gyration were calculated Fig. (7-9) and the results of RMSD showed that the peak started at $0.125 \mathrm{~nm}$ and the RMSD was not deviated above $0.25 \mathrm{~nm}$ during $10 \mathrm{~ns}$ simulation time. This indicated that the complex was stable. However, the RMSD was highly stable at 3 to $4 \mathrm{~ns}$ and 7 to $9 \mathrm{~ns}$ during the entire simulation time. In the RMSF peak, most of the residues were not fluctuated above $0.2 \mathrm{~nm}$. When the fluctuation of the active site residues and main chain atoms are low, conformational changes will be low and it results in a stable conformation (Priyadarshini et al., 2014). In our study, the residues between 500 to 600 were not fluctuated above $0.15 \mathrm{~nm}$ and most of the other residues were also not fluctuated above $0.3 \mathrm{~nm}$ indicating that the complex is stable. Radius of gyration $(\mathrm{Rg})$ was also used 
to find the stability of docked complex based on shape and conformation (Singh et al., 2018) and for our docked complex also the $\mathrm{Rg}$ was stable from 2 to $6 \mathrm{~ns}$ and 6.5 to $8.5 \mathrm{~ns}$.

Hence, the present study concludes that Neoandrographolide from Andrographis paniculata, Hexahydrocurcumin from Zingiber officinale and Nirphyllin from Phyllanthus amarus are having the potential ability to act as inhibitors for the target protein Glycoprotein. Among these, Neoandrographolide from Andrographis paniculata is found to have the potential to act as the best inhibitor for Nipah Virus based on our preliminary studies. Nevertheless, further in vitro and in vivo studies are planned to be done to confirm the in silico findings of this study which might play a crucial role in the control of $\mathrm{NiV}$ infections in the days to come.

\section{Acknowledgements}

The authors thank the Department of Biotechnology (DBT), Government of India, New Delhi for providing facility and financial support to this work under the scheme of BTISNet (No.BT/BI/13/035/2017). Further, the authors thank Tamil Nadu Veterinary and Animal Sciences University, Chennai, Tamil Nadu, India and the Dean, Madras Veterinary College, Chennai, India for providing required facilities to complete this work successfully.

\section{References}

Ali, MH., S. Anwar, P. Kumar Roy and Ashrafuzzaman, M. 2018. Virtual Screening for Identification of Small Lead Compound Inhibitors of Nipah Virus Attachment Glycoprotein. J Pharmacogenomics Pharmacoproteomics. 09(02).

Archana, S., S. Chawla, M. Bhavya, M. Ramya, S. Anushree and Sathyamurthy, B. 2018. In silico studies on dengue and Nipah viral proteins with selected Azadirachta indica leaves constituents. World J Adv Health Res. 2: 88-97.

Bishop, K., and Broder, C. 2008. Hendra and Nipah Viruses: Lethal Zoonotic Paramyxoviruses, In: Emerging Infections (Eds.) W. Scheld, S. Hammer and J. Hughes. ASM Press, Washington DC. Pp. 155-87.

Chong, H.T., A. Kamarulzaman, C.T. Tan, K.J. Goh, T. Thayaparan, S.R. Kunjapan et al., 2001. Treatment of acute Nipah encephalitis with ribavirin. Ann Neurol. 49(6): 810-3.

Eaton, BT., C.C. Broder, D. Middleton and Wang, L.F. 2006. Hendra and Nipah viruses: different and dangerous. Nat Rev Microbiol. 4(1): 23-35.

Essmann, U., L. Perera, M.L. Berkowitz, T. Darden, H. Lee and Pedersen, L.G. 1995. A smooth particle mesh Ewald method. J Chem Phys. 103(19): 8577-93.

Horvath, C.M., R.G. Paterson, M.A. Shaughnessy, R. Wood, and Lamb, R.A.1992. Biological activity of paramyxovirus fusion proteins: factors influencing formation of syncytia. $J$ Virol. 66: 4564-4569.

Hsu, V.P., M.J. Hossain, U.D. Parashar, M.M. Ali, T.G. Ksiazek, I. Kuzmin et al., 2004. Nipah virus encephalitis reemergence, Bangladesh. Emerg Infect Dis. 10(12): 2082-7.

Luby, S.P., E.S. Gurley and Hossain, M.J. 2012. Transmission of human infection with Nipah virus, In: Institute of Medicine (US). Improving Food Safety through a One Health Approach: Workshop Summary (A11). National Academies Press (US): Washington (DC).

Mehmood, M.A., U. Sehar and Ahmad, N. 2014. Use of Bioinformatics Tools in Different Spheres of Life Sciences. J. Data Mining Genomics Proteomics. 5: 1000158.

Mohanraj, K., B.S. Karthikeyan, R.P. VivekAnanth, R.P.B. Chand, S.R. Aparna, P. Mangalapandi et al., 2018. IMPPAT: A curated database of Indian Medicinal Plants, Phytochemistry and Therapeutics. Sci Rep. 8(1): 1-17.

Moll, M., S. Diederich, H.D. Klenk, M. Czub and Maisner, A. 2004. Ubiquitous Activation of 
the Nipah Virus Fusion Protein Does Not Require a Basic Amino Acid at the Cleavage Site. J Virol. 78(18): 9705-12.

Moller-Tank, S., and Maury, W. 2015. Ebola Virus Entry: A Curious and Complex Series of Events. PLoS Pathog. 11(4): 1-8.

Montgomery, J.M., M.J. Hossain, E. Gurley, D.S. Carroll, A. Croisier, E. Bertherat, et al., 2008. Risk factors for Nipah virus encephalitis in Bangladesh. Emerg Infect Dis. 14(10): 1526-32.

Nor, M.M., C.H. Gan and Ong, B.L. 2000. Nipah virus infection of pigs in peninsular Malaysia Epidemiological findings. Rev Sci Tech. 19(1): 160-5.

Priyadarshini, V., D. Pradhan, M. Munikumar, S. Swargam, A. Umamaheswari and Rajasekhar, D. 2014. Genome-based approaches to develop epitope-driven subunit vaccines against pathogens of infective endocarditis. J Biomol Struct Dyn. 32(6): 876-89.

Schuttelkopf, A.W., and Van Aalten, D.M.F. 2004. PRODRG: A tool for highthroughput crystallography of proteinligand complexes. Acta Crystallogr Sect D Biol Crystallogr. 60(8): 1355-63.

Searo (South-East Asia Regional Office): Emerging diseases, 2019. http://www.searo.who.int/entity/emerging _ diseases/en/. Accessed 5 June 2019b.

Searo (South-East Asia Regional Office): Nipah virus $\quad 2019$. http://www.searo.who.int/entity/emerging diseases/links/nipah_virus_outbreaks_sear/e n/.Accessed 7 June 2019a.

Shimojima, M., A. Takada, H. Ebihara, G. Neumann, K. Fujioka, T. Irimura et al., 2006. Tyro3 Family-Mediated Cell Entry of Ebola and Marburg Viruses. J Virol. 80(20): 10109-16.

Singh, S.P., N.I. Singh, K. Nongalleima, P. Doley, C.B. Singh and Sahoo, D. 2018. Molecular docking, MD simulation, DFT and ADMEtoxicity study on analogs of zerumbone against IKK- $\beta$ enzyme as anti-cancer agents. Netw Model Anal Heal Informatics Bioinforma. 7(1).

Van Der Spoel, D., E. Lindahl, B. Hess, G. Groenhof, A.E. Mark and Berendsen, H.J.C. 2005. GROMACS: Fast, flexible, and free. J Comput Chem. 26(16): 1701-18.

Wang, L.F., B.H. Harcourt, M. Yu, A. Tamin, P.A. Rota, W.J. Bellini et al.2001. Molecular biology of Hendra and Nipah viruses. Microbes Infect. 3(4): 279-87.

WHO: Nipah 2019. https://www.who.int/news-room/factsheets/detail/nipah-virus.Accessed 16 May 2019.

WOAH (World Organisation for Animal Health) (Office International des Epizooties: OIE). 2009. Nipah virus encephalitis. Technical Disease Cards, OIE: Paris.

\section{How to cite this article:}

Raja, T., P. Ravikumar, M. R. Srinivasan, K. Vijayarani and Kumanan, K. 2020. Identification of Potential Novel Inhibitors for Nipah Virus - An in silico Approach. Int.J.Curr.Microbiol.App.Sci. 9(09): 3377-3390. doi: https://doi.org/10.20546/ijcmas.2020.909.420 